\title{
TANGENT 2-FIELDS ON EVEN-DIMENSIONAL NONORIENTABLE MANIFOLDS
}

\author{
BY
}

BENEDICT J. POLLINA

\begin{abstract}
This paper uses the Postnikov decomposition of a nonsimple fibration to describe the obstructions to a tangent 2-field on an even-dimensional nonorientable manifold.
\end{abstract}

Our purpose in this article is to prove the following theorem.

MaIN TheOREM. Let $M$ be a closed, nonorientable manifold of dimension $m$, where $m$ is even and $m>2$. Let $\mathscr{X}(M)$ in $H^{m}\left(M ; Z_{w_{1}(M)}\right)$ and $\mathcal{Q} \int_{m-1}(M)$ in $H^{m-1}\left(M ; Z_{w_{1}(M)}\right)$ denote the twisted Euler class of $M$ and the $(m-1)$ Stiefel-Whitney class of $M$, respectively. Then $M$ has a 2-field if, and only if, $\mathcal{X}(M)=0$ and Q $_{m-1}(M)=0$.

The vector field problem has a long history, and in the special case of 2 -fields a great deal is already known. In order to put this result in perspective it will be helpful to give a brief statement of the known results. For details and an extensive bibliography the reader is referred to the excellent survey paper by Emery Thomas [10].

Thomas [10], Frank [3], and Atiyah [1] have described completely the necessary and sufficient conditions for a closed orientable manifold to have a 2-field. Suppose $M$ is such a manifold of dimension $m$. Let $w_{m-1}(M)$ in $H^{m-1}\left(M ; Z_{2}\right)$ be the $(m-1)$ Stiefel-Whitney class, $X(M)$ the Euler class, $k(M)$ the real Kervaire semicharacteristic, and $\sigma(M)$ the signature of $M$. Their results may then be summarized as follows.

\begin{tabular}{cl}
$\begin{array}{c}\text { Dimension } \\
\text { of } M \text { mod } 4\end{array}$ & $\begin{array}{l}\text { Necessary and sufficient conditions } \\
\text { for a 2-field on } M\end{array}$ \\
\hline 3 & $M$ always has a 2-field \\
2 & $X(M)=0$ \\
1 & $w_{m-1}(M)=0$ and $k(M)=0$ \\
0 & $w_{m-1}(M)=0, X(M)=0$, and $\sigma(M) \equiv 0 \bmod 4$
\end{tabular}

Received by the editors July 24,1980 and, in revised form, February 6, 1981.

1980 Mathematics Subject Classification. Primary 57R25; Secondary 55S35, 55S45.

Key words and phrases. Tangent 2-field, Postnikov decomposition, nonsimple fibration, local system of integers. 
In addition, Atiyah and Dupont [2] have given necessary and sufficient conditions for a 2-field on a nonorientable $4 k+1$ manifold which satisfies $w_{1}^{2}(M)=0$. Their result is somewhat complicated, and we will not attempt to state it here.

A summary of nonsimple Postnikov theory. Postnikov theory in the nonsimple case has been developed extensively by McClendon [5], Siegel [7], and C. A. Robinson [6]. Before proceeding to the proof of the Main Theorem we summarize their results in a form which will be convenient for our application.

Let $F \rightarrow E \stackrel{p}{\rightarrow} B$ be a fibration which is not necessarily simple, that is, $\pi_{1}(B)$ may act nontrivially on $\pi_{*}(F)$. We will assume throughout that $F, E, B$ are all path connected and that $F$ is simply connected. In this way we get a natural action of $\pi_{1}(B)$ on the homotopy of $F$. Let $G$ be an abelian group and $h: \pi_{1}(B) \rightarrow \operatorname{Aut}(G)$ a homomorphism determining up to isomorphism a local coefficient system $G_{h}$ on $B$. Define a map $\tilde{h}: \pi_{1}(B) \rightarrow \operatorname{Aut}\left(H^{q}(F ; G)\right)$ in the following way. Given an element $b$ in $\pi_{1}(B), b$ determines an automorphism $\bar{b}: F \rightarrow F$ in the usual way. Let

$$
\bar{b}^{*}: H^{q}(F ; G) \rightarrow H^{q}(F ; G)
$$

be the induced isomorphism in cohomology. The element $b$ also determines an isomorphism $h(b): G \rightarrow G$ which induces a coefficient isomorphism

$$
h(b)_{*}: H^{q}(F ; G) \rightarrow H^{q}(F ; G) .
$$

Now define $\tilde{h}(b)$ to be the composite $h(b)_{*} \circ \bar{b}^{*}$. The map $\tilde{h}$ determines a new local system on $B$ denoted $H^{q}(F ; G)_{h}$. We now have the following "twisted Serre spectral sequence".

THEOREM 1 (SIEGEL [7]). There exists a cohomology spectral sequence $E_{r}^{p . q}$ such that

$$
E_{2}^{p, q}=H^{p}\left(B ; H^{q}(F ; G)_{\tilde{h}}\right), \quad E_{r}^{p, q} \Rightarrow H^{*}\left(E ; p^{-1} G_{h}\right) .
$$

Remarks. $p^{-1} G_{h}$ denotes the local coefficient system on $E$ induced by the map $p: E \rightarrow B$. McClendon [5] has given a relative version of this theorem.

From this spectral sequence one obtains the following results.

Corollary 1. Let $H^{p}\left(B ; H^{q}(F ; G)_{n}\right)=0$ whenever $0<q<n$ or $0<p<m$. Then there is an exact sequence

$$
\begin{aligned}
\cdots \rightarrow H^{k}\left(B ; G_{h}\right) & \stackrel{p^{*}}{\rightarrow} H^{k}\left(E ; p^{-1} G_{h}\right) \stackrel{i^{*}}{\rightarrow}\left[H^{k}(F ; G)\right]^{\tilde{h}} \\
& \stackrel{\tau}{\rightarrow} H^{k+1}\left(B ; G_{h}\right) \rightarrow \cdots \rightarrow H^{m+n-1}\left(E ; p^{-1} G_{h}\right) .
\end{aligned}
$$

The group

$$
\left[H^{k}(F ; G)\right]^{\tilde{h}}=\left\{a \in H^{k}(F ; G) \mid \tilde{h}(a)=\text { a for all } a \text { in } \pi_{1}(B)\right\},
$$

that is, the subgroup of elements invariant under the action $\tilde{h}$.

Corollary 2. Assume $H^{p}\left(B ; H^{q}(F ; G) \tilde{h}\right)=0$ for $0<q<n$. Then

$$
p^{*}: H^{i}\left(B ; G_{h}\right) \rightarrow H^{i}\left(E ; p^{-1} G_{h}\right)
$$

is an isomorphism for $i<n$, and the following sequence is exact. 


$$
\begin{aligned}
0 \rightarrow H^{n}\left(B ; G_{h}\right) & \stackrel{p^{*}}{\rightarrow} H^{n}\left(E ; p^{-1} G_{h}\right) \stackrel{i^{*}}{\rightarrow}\left[H^{n}(F ; G)\right]^{\tilde{h}} \\
& \stackrel{\tau}{\rightarrow} H^{n+1}\left(B ; G_{h}\right) \stackrel{p^{*}}{\rightarrow} H^{n+1}\left(E ; p^{-1} G_{h}\right) .
\end{aligned}
$$

These sequences will be important later on when we construct the Postnikov tower for the map $p$.

Next we consider classifying spaces for twisted cohomology. We will confine our attention to twisted integer cohomology, since this will suffice for our applications. A much more general treatment, however, is possible. Let $h: Z_{2} \rightarrow \operatorname{Aut}(Z)$ be the nontrivial action of $Z_{2}$ on $Z$, and let $Z_{2} \rightarrow \tilde{K} \rightarrow K\left(Z_{2}, 1\right)$ be the universal cover. A space of type $K(Z, n)$ can be constructed so that $h$ determines an action $\hat{h}$ of $Z_{2}$ on $K(Z, n)$ by basepoint preserving homeomorphisms. Take the Cartesian product $\tilde{K} \times K(Z, n)$, and let $Z_{2}$ act by covering transformations on the first factor and by $\hat{h}$ on the second. Denote the quotient space of this action by $L(Z, n)$. Clearly, one has a fibration

$$
K(Z, n) \stackrel{i}{\rightarrow} L(Z, n) \stackrel{p}{\rightarrow} K\left(Z_{2}, 1\right)
$$

Furthermore, since the action of $Z_{2}$ on $K(Z, n)$ has a fixed point, the fibration has a canonical section $s: K\left(Z_{2}, 1\right) \rightarrow L(Z, n)$.

Let $X$ be a complex and $w: X \rightarrow K\left(Z_{2}, 1\right)$ a fixed map. The map $w$ determines a local system of integers on $X$, denoted $Z_{w}$. Let $[X, L(Z, n) ; w]$ denote the set of homotopy classes of lifts of $w$ into $L(Z, n)$, where the homotopies are required to go through lifts of $w$. Then one has the following result.

THEOREM 2. There is an element $e_{n}$ in $H^{n}\left(L(Z, n) ; Z_{p}\right)$ with the property that the mapping

$$
\begin{array}{ccc}
{[X, L(Z, n) ; w]} & \rightarrow & H^{n}\left(X ; Z_{w}\right) \\
f & \rightarrow & f^{*}\left(e_{n}\right)
\end{array}
$$

is a bijection.

The proof of this theorem can be found in Steenrod [8, 37.5]. It is not difficult to show that $s^{*}\left(e_{n}\right)=0$ and $i^{*}\left(e_{n}\right)=\iota_{n}$, where $\iota_{n}$ in $H^{n}(K(Z, n) ; Z)$ is the fundamental class.

Let $k_{0} \in K(Z, n)$ be the basepoint, and let $\operatorname{PK}(Z, n)$ be the space of paths in $K(Z, n)$ starting at $k_{0}$. Since the action $\hat{h}$ of $Z_{2}$ on $K(Z, n)$ fixes the basepoint, $Z_{2}$ acts on $P K(Z, n)$ in the obvious way. As before, we can form the space $\tilde{K} \times P K(Z, n)$ and take the quotient of the $Z_{2}$ action. The resulting space will be denoted $\bar{P} L(Z, n)$ and is the total space of a fibration:

$$
P K(Z, n) \rightarrow \bar{P} L(Z, n) \stackrel{\bar{p}}{\rightarrow} K\left(Z_{2}, 1\right) .
$$

Further, let $1 \times e: \tilde{K} \times P K(Z, n) \rightarrow \tilde{K} \times K(Z, n)$ be defined by $(1 \times e)(\tilde{k}, a)=$ $(\tilde{k}, a(1))$. This map is a fibration with fibre $\Omega K(Z, n)$, and it is easily checked that $1 \times e$ is $Z_{2}$ equivariant. Thus $1 \times e$ induces a map $\bar{e}: \bar{P} L(Z, n) \rightarrow L(Z, n)$ which is 
also a fibration with fibre $\Omega K(Z, n)$. We can summarize the above constructions in the following commutative diagram.

$$
\begin{array}{ccccc}
K(Z, n-1) & \rightarrow & P K(Z, n) & \stackrel{e}{\rightarrow} & K(Z, n) \\
\| & & \downarrow & & \downarrow \\
K(Z, n-1) \rightarrow & \bar{P} L(Z, n) & \stackrel{\bar{e}}{\rightarrow} & L(Z, n) \\
& & \downarrow \bar{p} & & \downarrow p \\
& & K\left(Z_{2}, 1\right) & = & K\left(Z_{2}, 1\right)
\end{array}
$$

Note that we have used the fact that $\Omega K(Z, n)$ has the same homotopy type as $K(Z, n-1)$.

There is one last space we will need. Let $Z_{2}$ act on $\Omega K(Z, n)$ in the manner analogous to the above and form the space $\bar{\Omega} L(Z, n)$. As before this space fibres over $K\left(Z_{2}, 1\right)$ with fibre $\Omega K(Z, n)$. It is easy to show that $\bar{\Omega} L(Z, n)$ has the same homotopy type as $L(Z, n-1)$.

Next we consider the analogue of a principal fibration in ordinary Postnikov theory. Let $B$ be a space with a local system of integers $Z_{w}$ determined by a map $w: B \rightarrow K\left(Z_{2}, 1\right)$. Let $v$ be an element of $H^{n+1}\left(B ; Z_{w}\right)$. Consider the diagram

$$
\begin{array}{ccccc}
K(Z, n) \stackrel{i}{\rightarrow} & E & \stackrel{\bar{c}}{\rightarrow} & \bar{P} L(Z, n+1) \\
& \downarrow p_{1} & & \downarrow \bar{e} \\
& B & \stackrel{v}{\rightarrow} & L(Z, n+1) .
\end{array}
$$

The fibration $p_{1}$ is the pullback by $v$ of $\bar{e}$. Thus

$$
E=\{(b, u) \in B \times \bar{P} L(Z, n) \mid v(b)=\bar{e}(u)\} .
$$

Observe that $E$ maps to $K\left(Z_{2}, 1\right)$ by the composite $w p_{1}$. This defines an induced local system of integers on $E$. Define the space

$$
E \oplus \bar{\Omega} L(Z, n+1)=\{((b, u), s) \in E \times \bar{\Omega} L(Z, n+1) \mid w(b)=p(s)\},
$$

where $p$ denotes the fibration $p: \bar{\Omega} L(Z, n+1) \rightarrow K\left(Z_{2}, 1\right)$. Define a map

$$
\mu: E \oplus \bar{\Omega} L(Z, n+1) \rightarrow E \text { by } \mu((b, u), s)=(b, s+u) .
$$

This map is the analogue of the principal action map for ordinary principal fibrations in the following sense. Suppose $X$ is a complex, and $q: X \rightarrow B$ is a given map. Let $f, g: X \rightarrow E$ be two lifts of $q$, so that $p_{1} f \simeq p_{1} g \simeq q$. Define the space

$$
X \oplus X=\left\{\left(x, x^{\prime}\right) \in X \times X \mid w q(x)=w q\left(x^{\prime}\right)\right\} .
$$

Proposition. There exists a map $d: X \rightarrow \bar{\Omega} L(Z, n+1) \cong L(Z, n)$ such that the composite

$$
X \stackrel{\Delta}{\rightarrow} X \oplus X^{f \oplus d} \rightarrow E \oplus \bar{\Omega} L(Z, n+1) \stackrel{\mu}{\rightarrow} E
$$


is homotopic to $g$. Moreover $d$ satisfies the property that $p d=w q$, that is, the diagram

$$
\begin{array}{lll}
X & \stackrel{d}{\rightarrow} & \bar{\Omega} L(Z, n+1) \\
& & \\
& & \swarrow p \\
& K\left(Z_{2}, 1\right) &
\end{array}
$$

commutes. Thus, by the identification

$$
\bar{\Omega} L(Z, n+1) \cong L(Z, n),
$$

we have $d \in H^{n}\left(X ; q^{-1} Z_{w}\right)$.

COROLlaRY. If $d$ represents the zero element of $H^{n}\left(X ; q^{-1} Z_{w}\right)$, then $f$ is homotopic to g.

The map $\mu: E \oplus \bar{\Omega} L(Z, n+1) \rightarrow E$ also has an inverse $T$ defined by $T(b, s)=$ $((b, s), c)$ where $b \in B, s \in \bar{P} L(Z, n)$, and $c$ is the constant path in $K(Z, n+1)$ over the point $w(b)$. It is not difficult to see that $\mu T \simeq 1_{E}$.

Having given a cursory description of the construction and properties of the spaces required for nonsimple Postnikov theory, we are in a position to describe the actual tower for the fibration $F \rightarrow E \stackrel{p}{\rightarrow} B$. To do this we need the following result.

TheOREM 3 (MCClendon [5]). Assume $F$ is $(n-1)$ connected for $n \geqslant 2$. Let $\mathrm{t}$ in $H^{n}\left(F ; \pi_{n}(F)\right)$ be the fundamental class and $h: \pi_{1}(B) \rightarrow \operatorname{Aut}\left(\pi_{n}(F)\right)$ the local system defined by the fibration. Then $\mathrm{c}$ is in $\left[H^{n}\left(F ; \pi_{n}(F)\right)\right]^{h}$, and, hence, by Corollary 2 to Theorem 1, it is transgressive.

The Postnikov tower for $F \rightarrow E \rightarrow B$ can now be constructed. Let $\iota$ in $H^{n}\left(F ; \pi_{n}(F)\right)$ be the fundamental class of $F$, and let $h: \pi_{1}(B) \rightarrow \operatorname{Aut}\left(\pi_{n}(F)\right)$ be the local system on $B$ defined by the fibration. By the previous theorem, $\tau(\iota)=k$, is defined and is an element of $H^{n+1}\left(B ; \pi_{n}(F)_{h}\right)$. Construct the diagram

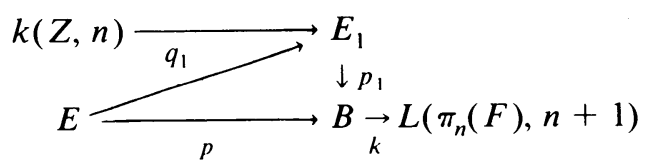

$E_{1}$ is the total space of the fibration which is the pullback by $k$ of the fibration $\bar{P} L\left(\pi_{n}(F), n+1\right) \rightarrow L\left(\pi_{n}(F), n+1\right)$. Since $\tau(\iota)=k$, by exactness we must have $p^{*}(k)=0$. Therefore, there exists a map $q_{1}: E \rightarrow E_{1}$ such that $p_{1} q_{1}=p$. By a standard argument, the fibre $F_{1}$ of $q_{1}$ is $n$-connected and $\pi_{k}\left(F_{1}\right)=\pi_{k}(F)$ for $k>n$. The space $E_{1}$ is the second stage of the Postnikov tower. To construct higher stages the above construction is iterated on the successive fibrations $q_{i}$.

Proof of the Main Theorem. We begin by constructing a Postnikov tower for the fibration

$$
V_{m, 2} \rightarrow B O(m-2) \stackrel{p}{\rightarrow} B O(m)
$$

where $m$ is even, and $V_{m, 2}$ is the Stiefel manifold of 2-frames in euclidean $m$ space. The fibre $V_{m, 2}$, hereafter denoted simply by $V$, is $(m-3)$ connected; $\pi_{m-2}(V)=Z$, 
and $\pi_{m-1}(V)=Z \oplus Z_{2}$. The action of $\pi_{1} B O(n)=Z_{2}$ is nontrivial on $\pi_{m-2}(V)$ and on the $Z$-summand of $\pi_{m-1}(V)$.

Lemma 1. Let $\iota$ in $H^{m-2}(V ; Z)$ be the fundamental class of $V$. Then $\tau(\iota)=\mathcal{O l}_{m-1}$ in $H^{m-1}\left(B O(m) ; Z_{w_{1}}\right)$.

Lemma 2. Let $\sigma: H^{m}\left(B O(m) ; Z_{w_{1}}\right) \rightarrow H^{m-1}(V ; Z)$ denote the suspension map for the fibration $p$. Then $\sigma(\chi)$ contains a spherical class.

REMARK. For the definition and properties of spherical classes the reader is referred to the article of McClendon [5].

These two lemmas imply that we may use $015_{m-1}$ and $\chi$ in the first stage of our Postnikov tower to kill off $\pi_{m-2}(V)$ and the $Z$-summand of $\pi_{m-1}(V)$. Thus we have the diagram

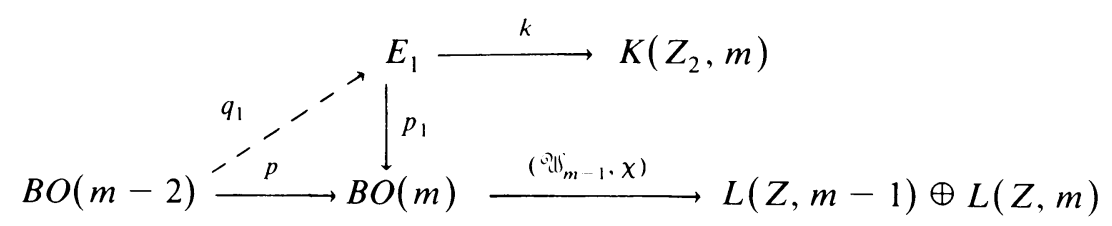

$L(Z, m-1) \oplus L(Z, m)$ denotes the fibre product over $K\left(Z_{2}, 1\right)$ of $L(Z, m)$ and $L(Z, m-1)$. The map $p$ lifts to a map $q_{1}: B O(m-2) \rightarrow E_{1}$, since $p^{*}(\chi)$ and $p^{*}\left(\left.\mathcal{Q}\right|_{m-1}\right)$ are both zero. Convert the lift $q_{1}$ into a fibration with fibre $F_{1}$. Then $F_{1}$ is $(m-2)$ connected, and $\pi_{m-1}\left(F_{1}\right)=Z_{2}$. Let $u \in H^{m-1}\left(F_{1} ; Z_{2}\right)$ be the fundamental class, and set $k=\tau(u)$ in $H^{m}\left(E_{1} ; Z_{2}\right)$. The element $k$ is the second Postnikov invariant for our fibration $p$. Let $K\left(Z_{2}, m-1\right) \rightarrow E_{2} \stackrel{p_{2}}{\rightarrow} E_{1}$ be the principal fibration induced by $k$. We then have the following diagram.

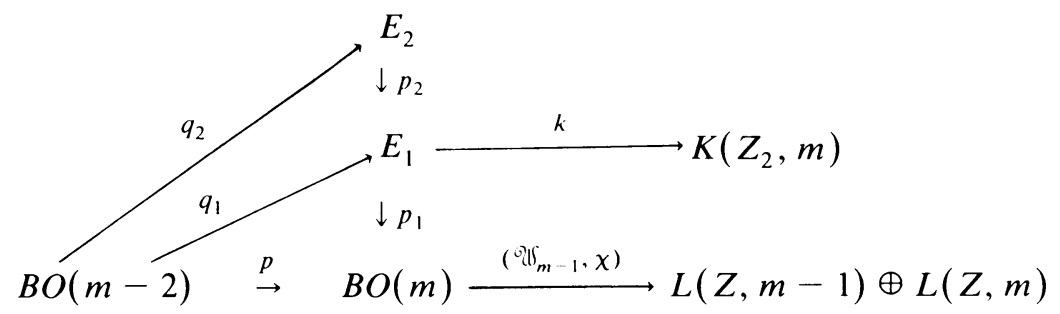

The fibre $F_{2}$ of $q_{2}$ is $(m-1)$ connected, and therefore by a standard argument the map $q_{2 \sharp}:[X, B O(m-2)] \rightarrow\left[X, E_{2}\right]$ is a bijection for a complex $X$ of dimension less than or equal to $m$. Thus if we are given a map $f: X \rightarrow B O(m), f$ lifts to $g$ : $X \rightarrow B O(m-2)$ if, and only if, $f$ lifts to $E_{2}$. If $f^{*}(\chi)=f^{*}\left(\mathcal{Q} \int_{m-1}\right)=0$, then $f$ lifts to a map $g: X \rightarrow E_{1}$, and it is clear that $f$ lifts to $E_{2}$ if, and only if, we can choose a lift $g: X \rightarrow E_{1}$ for which $g^{*}(k)=0$. 
Lemma 3. Define $\bar{E}$ to be the space $E_{1} \oplus L(Z, m-2) \oplus L(Z, m-1)$, the fibre product over $K\left(Z_{2}, 1\right)$. Let $\mu: \bar{E} \rightarrow E_{1}$ be the map defined by $(*)$, and let $e_{m-2} \in$ $H^{m-2}\left(L(Z, m-2) ; Z_{p}\right)$ and $e_{m-1} \in H^{m-1}\left(L(Z, m-1) ; Z_{p}\right)$ be the twisted fundamental classes of Theorem 2 . Then we have the following formula.

$$
\begin{aligned}
\mu^{*}(k)= & k \otimes 1 \otimes 1+1 \otimes \mathrm{Sq}^{1} e_{m-1} \otimes 1+1 \otimes 1 \\
& \otimes \mathrm{Sq}^{2} e_{m-2}+w_{2} \otimes 1 \otimes e_{m-2} .
\end{aligned}
$$

The proofs of Lemmas 1-3 will be given later.

COROLlary. If $g_{1}$ and $g_{2}$ are two lifts of $f$ to $E_{1}$, then there exist elements $u_{1}$ in $H^{m-1}\left(X ; Z_{f^{*}\left(w_{1}\right)}\right)$ and $u_{2} \in H^{m-2}\left(X ; Z_{f^{*}\left(w_{1}\right)}\right)$ such that

$$
g_{2}^{*}(k)=g_{1}^{*}(k)+\mathrm{Sq}^{1} u_{1}+\mathrm{Sq}^{2} u_{2}+f^{*}\left(w_{2}\right) u_{2} .
$$

Proof. Use Lemma 3 and the Proposition.

The corollary shows that the elements $g^{*}(k)$ as $g$ runs over lifts of $f$ to $E_{1}$ form a coset in $H^{m}\left(X ; Z_{2}\right)$ of the subgroup

$$
\mathrm{Sq}^{1} H^{m-1}\left(X ; Z_{f^{*}\left(w_{1}\right)}\right)+\left(\mathrm{Sq}^{2}+f^{*}\left(w_{2}\right)\right) H^{m-2}\left(X ; Z_{f^{*}\left(w_{1}\right)}\right) .
$$

This immediately gives us the following theorem.

THEOREM 4. Let $X$ be a complex of dimension $m$, where $m$ is even. Suppose $f$ : $X \rightarrow B O(m)$ satisfies $f^{*}\left(\mathcal{W}_{m-1}\right)=0$ and $f^{*}(\chi)=0$. Then flifts to $f: X \rightarrow B O(m-2)$ if, and only if, $g^{*}(k)=0$ in

$$
H^{m}\left(X ; Z_{2}\right) / \mathrm{Sq}^{1} H^{m-1}\left(X ; Z_{f^{*}\left(w_{1}\right)}\right)+\left(\mathrm{Sq}^{2}+f^{*}\left(w_{2}\right)\right)\left(H^{m-2}\left(X ; Z_{f^{*}\left(w_{1}\right)}\right)\right)
$$

where $g$ is any lift of $f$ to $E_{1}$.

The Main Theorem is now an easy consequence of the following lemma.

Lemma 4. Let $M$ be a nonorientable manifold of dimension $m$. The map $\mathrm{Sq}^{1}$ : $H^{m-1}\left(M ; Z_{w_{1}(M)}\right) \rightarrow H^{m}\left(M ; Z_{2}\right)$ is onto.

Proof. We have the following commutative triangle:

$$
\begin{array}{rcr}
H^{m-1}\left(M ; Z_{w_{1}(M)}\right) & \stackrel{\mathrm{Sq}^{1}}{\rightarrow} \quad H^{m}\left(M ; Z_{2}\right) \\
\rho_{2} \searrow & \multicolumn{2}{c}{\mathrm{Sq}^{1}} \\
& H^{m-1}\left(M ; Z_{2}\right)
\end{array}
$$

where $\rho_{2}$ is reduction mod 2. It is well known that $\mathrm{Sq}^{1}: H^{m-1}\left(M ; Z_{2}\right) \rightarrow H^{m}\left(M ; Z_{2}\right)$ is onto. But $\rho_{2}$ is also onto as can be seen from the following exact sequence:

$$
\cdots \rightarrow H^{m-1}\left(M ; Z_{w_{1}(M)}\right) \stackrel{\rho_{2}}{\rightarrow} H^{m-1}\left(M ; Z_{2}\right) \stackrel{\beta}{\rightarrow} H^{m}\left(M ; Z_{w_{1}(M)}\right) \rightarrow \cdots,
$$

where $\beta$ is the Bockstein homomorphism associated to the coefficient sequence $0 \rightarrow Z_{w_{1}} \rightarrow Z_{w_{1}} \rightarrow Z_{2} \rightarrow 0$. Since $H^{m}\left(M ; Z_{w_{1}(M)}\right)$ is infinite cyclic and $H^{m-1}\left(M ; Z_{2}\right)$ 
has order 2 , we immediately see that $\beta$ is zero, and hence $\rho_{2}$ is onto. Thus $\mathrm{Sq}^{1}$ : $H^{m-1}\left(M ; Z_{w_{1}(M)}\right) \rightarrow H^{m}\left(M ; Z_{2}\right)$ is the composition of onto maps and must itself be onto.

To prove the Main Theorem let $f: M \rightarrow B O(m)$ be a map classifying $T(M)$, the tangent bundle of $M$. By hypothesis, $f^{*}(\chi)$ and $f^{*}\left(\mathcal{O} \int_{m-1}\right)$ are zero. Choose any lift of $f$ to the space $E_{1}$; call it $g$. Then $g^{*}(k)$ is an element of the quotient group

$$
H^{m}\left(M ; Z_{2}\right) / \mathrm{Sq}^{1} H^{m-1}\left(M ; Z_{w_{1}(M)}\right)+\left(\mathrm{Sq}^{2}+w_{2}(M)\right) H^{m-2}\left(M ; Z_{w_{1}(M)}\right) .
$$

But this group is zero by Lemma 4 , and so by Theorem $4 f$ lifts to $B O(m-2)$.

It only remains for us to prove Lemmas 1 through 3.

Proof of Lemma 1. Corollary 2 to Theorem 1 gives us the following exact sequence.

$$
\rightarrow\left[H^{m-2}(V ; Z)\right]^{\bar{h} \tau} \rightarrow H^{m-1}\left(B O(m), Z_{w_{1}}\right) \stackrel{p^{*}}{\rightarrow} H^{m-1}\left(B O(m-2) ; Z_{w_{1}}\right) \rightarrow .
$$

The group on the left is $Z$, generated by $\iota$ according to Theorem 3 . The kernel of $p^{*}$ is generated by $\mathscr{Q} \int_{m-1}$, so by exactness $\tau(\iota)=\mathscr{Q} \int_{m-1}$.

Proof of Lemma 2. Consider the diagram

$$
\begin{array}{ccccc}
V_{m, 2} & \rightarrow & B O(m-2) & \stackrel{p}{\rightarrow} & B O(m) \\
g \downarrow & & \downarrow & & \| \\
S^{m-1} & \rightarrow & B O(m-1) & \stackrel{p^{\prime}}{\rightarrow} & B O(m)
\end{array}
$$

Let $\sigma$ be the suspension for $p$ and $\sigma^{\prime}$ the suspension for $p^{\prime}$. By the Serre and Gysin sequences for $p^{\prime}$ it is easy to see that $\sigma^{\prime}(\chi)=v$, where $v$ is a generator of $H^{m-1}\left(S^{m-1} ; Z\right)$. So $\sigma^{\prime}(\chi)$ contains a spherical class, namely $v$. The map $g$ is a fibration with fibre $S^{m-2}$. Consider the homotopy exact sequence for $g$ :

$$
\rightarrow \pi_{m-1}\left(V_{m, 2}\right) \stackrel{g^{*}}{\rightarrow} \pi_{m-1}\left(S^{m-1}\right) \stackrel{\Delta}{\rightarrow} \pi_{m-2}\left(S^{m-2}\right) \stackrel{i^{*}}{\rightarrow} \pi_{m-2}\left(V_{m, 2} \stackrel{g^{*}}{\rightarrow} \pi_{m-2}\left(S^{m-1}\right) .\right.
$$

Since $\pi_{m-2}\left(S^{m-1}\right)$ is zero, we must have $i_{*}$ onto. But $\pi_{m-2}\left(S^{m-2}\right)$ and $\pi_{m-2}\left(V_{m, 2}\right)$ are both infinite cyclic, and consequently $i_{*}$ is also 1-1. By exactness, this makes $g_{*}$ : $\pi_{m-1}\left(V_{m-2}\right) \rightarrow \pi_{m-1}\left(S^{m-1}\right)$ an epimorphism. This implies that $g^{*}(v)$ in $\sigma(\chi)$ is spherical.

Proof of Lemma 3. Consider the following diagram:

$$
\begin{array}{ccc}
B O(m-2) \oplus L(Z, m-2) \oplus L(Z, m-1) & \stackrel{q_{1} \oplus 1 \oplus 1}{\rightarrow} & \bar{E} \stackrel{\mu}{\rightarrow} E_{1} \\
\bar{s} \uparrow \downarrow \pi & & \downarrow p_{1} \\
B O(m-2) & \stackrel{p}{\rightarrow} & B O(m)
\end{array}
$$

Recall that $\bar{E}=E_{1} \oplus L(Z, m-2) \oplus L(Z, m-1)$, the fibre product over $K\left(Z_{2}, 1\right)$. It is easy to see that the map $\pi$ has a section $\bar{s}$. Furthermore, if we let $v$ be the 
composite $\mu \circ\left(q_{1} \oplus 1 \oplus 1\right)$, then $v \bar{s} \simeq q_{1}$. By the work of Emery Thomas [9], there exist a map $\tau_{1}$ and a short exact sequence in $Z_{2}$ cohomology

$$
\begin{aligned}
0 & \rightarrow H^{m}\left(E_{1}\right) \stackrel{v^{*}}{\rightarrow} H^{m}(B O(m-2) \oplus L(Z, m-2) \oplus L(Z, m-1)) \\
& \stackrel{\tau_{1}}{\rightarrow} H^{m+1}(B O(m)) .
\end{aligned}
$$

Moreover, $\tau_{1}$ commutes with Steenrod squares and is an $H^{*}\left(B O(n) ; Z_{2}\right)$ module map. As described earlier, there is a map $T: E_{1} \rightarrow \bar{E}$ such that $\mu T \simeq 1 \bar{E}$. Therefore,

$$
\mu^{*}(k)=k \otimes 1 \otimes 1+\sum_{j} a_{j} \otimes b_{j} \otimes c_{j}
$$

where $a_{j}$ is in $H^{*}\left(E_{1} ; Z_{2}\right), b_{j}$ is in $H^{*}\left(L(Z, m-2) ; Z_{2}\right)$, and $c_{j}$ is in

$$
H^{*}\left(L(Z, m-1) ; Z_{2}\right)
$$

with $\operatorname{deg} a_{j}+\operatorname{deg} b_{j}+\operatorname{deg} c_{j}=m$ and $\operatorname{deg} b_{j}+\operatorname{deg} c_{j}>0$. This last condition implies that $\Sigma a_{j} \otimes b_{j} \otimes c_{j}$ is in $\operatorname{ker} \bar{s}^{*}$. The second Postnikov invariant $k$ is in $\operatorname{ker} q_{1}^{*}$, so $v^{*}(k)=\Sigma a_{j} \otimes b_{j} \otimes c_{j}$ is in $\operatorname{ker} \bar{s}^{*} \cap \operatorname{ker} \tau_{1}$ by the short exact sequence above.

Lemma 5. The intersection of $\operatorname{ker} \bar{s}^{*}$ with $H^{m}\left(B O(m-2) \oplus\left(L(Z, m-1) ; Z_{2}\right)\right)$ consists of the elements $1 \otimes \mathrm{Sq}^{1} e_{m-1} \otimes 1,1 \otimes 1 \otimes \mathrm{Sq}^{2} e_{m-2}, w_{1} \otimes 1 \otimes \mathrm{Sq}^{1} e_{m-2}$, and $w_{2} \otimes 1 \otimes e_{m-2}$.

Proof. Consider the mod 2 Serre spectral sequence for the fibration $\pi$ (see previous diagram). Since $\pi$ has a section, and since we are taking mod 2 cohomology, an easy argument shows that the spectral sequence collapses, that is, $E_{2}=E_{\infty}$. The conclusion of the lemma now follows easily.

We now compute the action of the map $\tau_{1}$ on these elements. Recall that by construction we have $\tau_{1}\left(1 \otimes e_{m-1} \otimes 1\right)=w_{m}$, where $w_{m}$ is the mod 2 reduction of $\chi$, and $\tau_{1}\left(1 \otimes 1 \otimes e_{m-2}\right)=w_{m-1}$. Using these formulas and those of $\mathrm{Wu}[11]$ we obtain

$$
\begin{aligned}
\tau_{1}\left(1 \otimes \mathrm{Sq}^{1} e_{m-1} \otimes 1\right) & =\mathrm{Sq}^{1} w_{m}=w_{1} w_{m}, \\
\tau_{1}\left(1 \otimes 1 \otimes \mathrm{Sq}^{2} e_{m-2}\right) & =\mathrm{Sq}^{2} w_{m-1}=w_{2} w_{m-1}+w_{1} w_{m}, \\
\tau_{1}\left(w_{1} \otimes 1 \otimes \mathrm{Sq}^{1} e_{m-2}\right) & =w_{1}^{2} w_{m-1}, \\
\tau_{1}\left(w_{2} \otimes 1 \otimes e_{m-2}\right) & =w_{2} w_{m-1} .
\end{aligned}
$$

Therefore, $\operatorname{ker} \tau_{1} \cap \operatorname{ker} \bar{s}^{*}$ must consist of the single element

$$
1 \otimes \mathrm{Sq}^{1} e_{m-1} \otimes 1+1 \otimes 1 \otimes \mathrm{Sq}^{2} e_{m-2}+w_{2} \otimes 1 \otimes e_{m-2} .
$$

By the short exact sequence given earlier, this gives us the required formula for $\mu^{*}(k)$.

ACKNOWLEDGEMENT. This article stems from research done for the author's doctoral dissertation. I wish to thank my advisor R. H. Szczarba for all his valuable assistance and Yale University for its generous financial support. My appreciation also to the University of Hartford for its support during the actual preparation of this paper. 


\section{REFERENCES}

1. M. F. Atiyah, Vector fields on manifolds, Arbeitsgemeinschaft für Forschung des Landes-NordrheinWestfalen, Heft 200, Westdeutsher Verlag, Koln, 1970.

2. M. F. Atiyah and J. L. Dupont, Vector fields with finite singularities, Acta Math. 128 (1972), 1-40.

3. D. Frank, On the index of a tangent 2-field, Topology 11 (1972), 245-252.

4. J. F. McClendon, Higher order twisted cohomology operations, Invent. Math. 7 (1969), 183-214.

5. __ Obstruction theory in fibre spaces, Math. Z. 120 (1971), 1-17.

6. C. A. Robinson, Moore-Postnikov systems for non-simple fibrations, Illinois J. Math. 16 (1972), 234-242.

7. J. Siegel, Higher order cohomology operations in local coefficient theory, Amer. J. Math. 89 (1967), 909-931.

8. N. Steenrod, The topology of fibre bundles, Princeton Univ. Press, Princeton, N. J., 1951.

9. E. Thomas, Seminar on fibre spaces, Lecture Notes in Math., vol. 13, Springer-Verlag, Berlin and New York, 1966.

10. __ Vector fields on manifolds, Bull. Amer. Math. Soc. 75 (1969), 643-683.

11. W. J. Wu, Classes-caracteristique et i-carrés d'une varieté, C. R. Acad. Sci. Paris 230 (1950), $508-511$.

Department of Mathematics and Physics, University of Hartford, West Hartford, ConNECTICUT 06117 